\title{
Optimal Control and Design of Electrical Machines
}

\author{
Wissem BEKIR ${ }^{1}$ \\ Research Laboratory Smart Electricity and ICT \\ SEICT, LR18ES44 \\ National Engineering School of Carthage \\ Université de Carthage \\ Tunis, Tunisia \\ Univ. Lille, Arts et Metiers \\ ParisTech, Centrale Lille, HEI, EA \\ 2697, L2EP, F-59000 \\ Lille, France
}

\author{
Lilia EL AMRAOUI ${ }^{2}$ \\ Research Laboratory Smart Electricity and ICT \\ SEICT, LR18ES44 \\ National Engineering School of Carthage \\ Université de Carthage, Tunis, Tunisia \\ Frédéric GILLON ${ }^{3}$ \\ Univ. Lille, Arts et Metiers \\ ParisTech, Centrale Lille, HEI, EA \\ 2697, L2EP, F-59000, Lille, France
}

\begin{abstract}
This paper presents a global optimization approach aiming to improve the energy efficiency of electrical machines. The process is made on a hybrid stepper motor allowing to simultaneously optimize design and command. This approach is axed around Pontryagin's maximum principle, which is applied to a magnetodynamic model based on permeances network model. The originality of the proposed approach is to obtain in the same process, the minimization of the energy by optimal control and the minimization of the energy by optimal sizing.
\end{abstract}

Keywords-Optimal control; optimal sizing; Pontryagin's maximum principle; permeances network; hybrid stepper motor; energetic efficiency

\section{INTRODUCTION}

Currently, improving the energy efficiency of electric machines is a subject of high interest. Indeed electrical machines are widely used in industries, transportation and home applications. Thus, electric machines consume the largest amount of energy in the world (i.e. $46 \%$ of global consumption resulting in about 6040 megatonnes of CO2) $[1,2]$.

The energy efficiency problem is studied in two different areas that require different skills. Firstly, automaticians deal with this problem as an optimal control issue. They seek to find the optimal control that allows minimizing either the energy consumption, subject to some constraints on the control, and/or the performances. Among the performance constraints, we can mention the constraints on the torque or for displacement problems, the constraints of positioning and speed [3]. Thus, the optimal control theory, which is a part of applied mathematics and automatic, is used in dynamic operation of the machine to find the trajectory of the command. However, the machine models used in the automatic field are coarse models [4-7] that do not take into consideration the geometric design parameters or not fully the magnetic phenomena.

Secondly, design specialists tackle the energy efficiency problem as an optimal design issue. Therefore, they seek to find the optimal design to achieve the required performance while minimizing also energy consumption. Thus, the models used are more complex and take into account with more accuracy the magnetic phenomena [8], the nature of the materials and the design parameters [9]. However, the models are quite complicated, the number of design parameters may be high and the search for optimality is carried out through optimization algorithms. Nevertheless, it would be absurd to seek for the optimal design of an electric machine without studying his command. Therefore, the command is one more parameter that is added to the optimization problems [10]. To overcome this difficulty the machine is optimized for operating points and imposing a form of control [11]. For example, by imposing a sinusoidal current control for a given machine, the problem is to find the optimum amplitude and angle of the control to have a certain torque and speed for a given operating point. The solution of this type of problem requires significant computation time, because of the extended model and the optimization algorithms used. Therefore, the difficulty is major if it is to find the optimal value of the command at any time, and in this case, the calculation time will be dissuasive.

In this paper, a method is proposed to solve this difficult problem. The idea is to merge the two domains and to develop a global optimization approach for design and control by applying optimal control theory [12-16] and nonlinear optimization algorithms on magnetodynamic models of an electric machine. This work is applied on a hybrid stepper motor [17-22], to prove the feasibility of the approach on a realistic case.

The paper is organized as follow; in the first part, a magnetic model of the machine is developed based on a permeances network. This model is coupled thereafter to a dynamic model that describes the electrical and mechanical behavior of the motor. In the second part, an optimal control theory is implemented, based on Pontryagin's maximum principle to this coupled magnetodynamic model. At first, an optimal control problem is posed. Then the optimality conditions are exploited to conclude on a Hamiltonian model that presents a two point boundary value problem. After that, a numerical method is proposed to solve this problem. Finally, optimal controls are calculated for a positioning problem. Results are then compared with a classical control. In the third part, a global optimization approach is proposed. The link between the different models and the resolution loops are presented. Then a global optimization problem is proposed 
followed by a study on the design parameter influence on the energy consumption of the machine. Finally, the global optimization problem is solved and the results are discussed.

\section{ModÉlIZATION}

The study is applied to a two-phase hybrid stepper motor, with 1024 steps per revolution illustrated in Fig. 1. This motor is composed of two rings; each one has 50-tooth. The two rings are angularly offset by a tooth half step. They are interconnected by a permanent magnet. The stator has 8 plots each having $Z_{s}$ teeth. The motor flux distribution is threedimensional.

\section{A. Magnetic Model}

The magnetic model is based on a permeances network method, which consists in decomposing the magnetic device into a set of flux sources and passive elements. Fig. 2 shows a front view and a rear view of the MPPH. $\mathcal{P}_{\alpha}, \mathcal{P}_{\beta}, \mathcal{P}_{\alpha}$, and $\mathcal{P}_{\beta}$, represents the permeances between the different stator plots and the two rotor rings. As the structure is symmetrical, this model focuses on the half of the machine.

Fig. 3 shows the equivalent magnetic circuit. The reluctance of iron is assumed infinite and the magnet is modeled by an ideal flux source Fm. The phases are modeled by the flux sources $F_{\alpha}$ and $F_{\beta}$.

The magnetic circuit resolution aims to determine the flux flowing in the branches. Equations are performed using Kirchhoff's laws; the fluxes generated are multiplied by 2 to represent the entire machine and by the number of coil, $N_{s}$, to describe the flux seen by the coils. The analytical expressions of the phases flux fed by currents $I_{\alpha}$ and $I_{\beta}$ generated are given by:

$\Phi_{\alpha}=2\left(\mathcal{P}_{\alpha}+\mathcal{P}_{\alpha}^{\prime}\right) N_{s}^{2} I_{\alpha}+\left(\mathcal{P}_{\alpha}-\mathcal{P}_{\alpha}^{\prime}\right) N_{s} F_{m}$

$\Phi_{\beta}=2\left(\mathcal{P}_{\beta}+\mathcal{P}_{\beta}^{\prime}\right) N_{s}^{2} I_{\beta}+\left(\mathcal{P}_{\beta}-\mathcal{P}_{\beta}^{\prime}\right) N_{s} F_{m}$

The inductance $L_{\alpha}$ (resp. $L_{\beta}$ ) of the phases $\alpha$ (resp. $\beta$ ) is then deduced:

$L_{\alpha}=2\left(\mathcal{P}_{\alpha}+\mathcal{P}_{\alpha}^{\prime}\right) N_{s}^{2}$

$L_{\beta}=2\left(\mathcal{P}_{\beta}+\mathcal{P}_{\beta}^{\prime}\right) N_{s}^{2}$

As well as the mutual flux between the phases and the magnet which are expressed by:

$\varphi_{\alpha}=\left(\mathcal{P}_{\alpha}-\mathcal{P}_{\alpha}^{\prime}\right) N_{s} F_{m}$

$\varphi_{\beta}=\left(\mathcal{P}_{\beta}-\mathcal{P}_{\beta}^{\prime}\right) N_{s} F_{m}$

The magnetomotive force expression of a magnet as a function of the length of the magnet $l_{m}$ and the coercive field $H_{c}$ is given by:

$F_{m}=l_{m} H_{c}$

An analytic method is used to calculate air gap permenances in order to have a fairly fast model. The method aims to represent flux lines by tubes formed with straight lines and arcs and to calculate the permeance of each flux tube as a function of displacement. Calculations are performed for a tooth step, the displacement is assumed linear since the length of the angular displacement of a step is negligible in front of the rotor radius. Fig. 4 describes the tooth structure and the approximation of the flux tubes for a given position. In this figure, $g$ represents the gap length, $t_{\mathrm{w}}$ the width of a tooth, $l_{r}$ the ring length, $P_{i}$ the permeances of flux tubes and $x$ is the linear displacement.

A linear displacement $x=2 t_{w}$ is equivalent to a rotation of a mechanical angle $\theta=7.2^{\circ}$. In [22] the permeance expressions are available and the results of this method have been validated by finite element method.

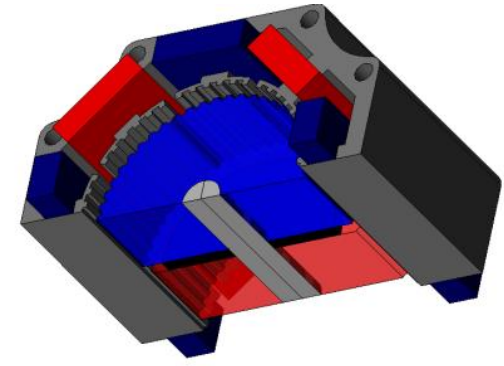

Fig. 1. Structure of the Hybrid Stepper Motor.

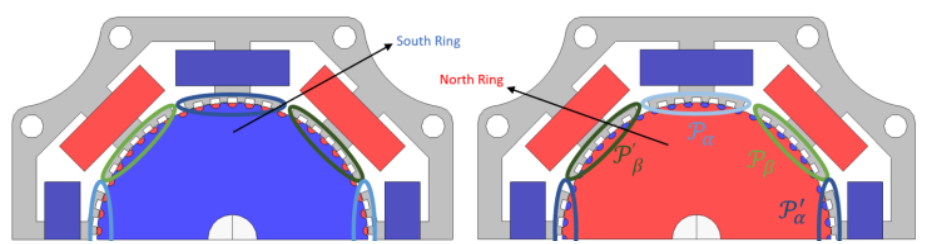

Fig. 2. HSM, Front and Rear View.

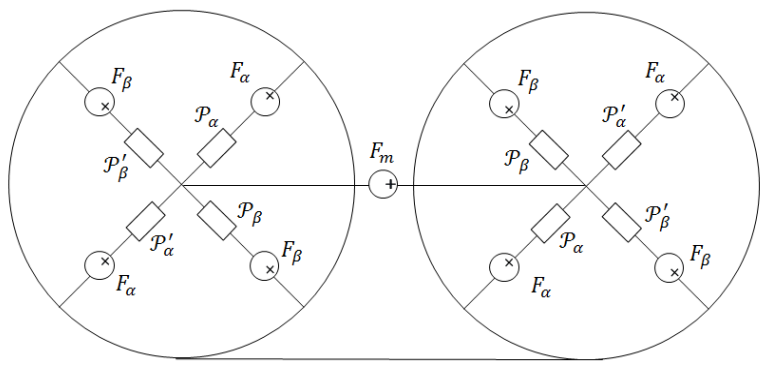

Fig. 3. Equivalent Magnetic Circuit.

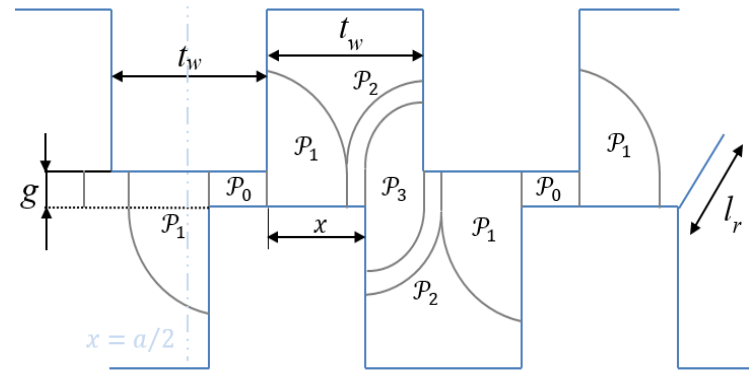

Fig. 4. Tooth Structure and the Approximation of the Flux Tubes. 


\section{B. Dynamic Model}

The equations of the voltages induced by the phases are expressed by:

$V_{\alpha}(t)=R I_{\alpha}(t)+I_{\alpha}(t) \frac{d L_{\alpha}(\theta)}{d t}+L_{\alpha}(\theta) \frac{d I_{\alpha}(t)}{d t}+\frac{d \varphi_{\alpha}(\theta)}{d t}$

$V_{\beta}(t)=R I_{\beta}(t)+I_{\beta}(t) \frac{d L_{\beta}(\theta)}{d t}+L_{\beta}(\theta) \frac{d I_{\beta}(t)}{d t}+\frac{d \varphi_{\beta}(\theta)}{d t}$

where $R$ is the resistance of the phases.

The fundamental principle of dynamics gives the following mechanical equation of motion (10):

$J_{r} \frac{\partial^{2} \theta(t)}{\partial t^{2}}=C_{e m}(t)-C_{r}(t)$

With:

$J_{\mathrm{r}} \quad:$ The rotor inertia,

$C_{e m}$ : electromagnetic torque,

$C_{r}$ : resistant couple.

The inertia $J_{\mathrm{r}}$ is bounded to the ring length $l_{r}$ and the magnet length $l_{m}$ as expressed in equation (11).

$J_{r}=2 \rho \pi r_{r}^{4} l_{r}+\frac{1}{2} \rho_{m} \pi r_{m}^{4}$

With:

$\rho$ : Density of motor iron,

$\rho_{m}:$ density of neodymium magnet,

$r_{r}$ : rotor radius,

$r_{m}$ : magnet radius.

The fundamental principle of energy conservation allows to find the expression of the power absorbed and to deduce the expression of the electromagnetic torque (12):

$C_{e m}(t)=\frac{1}{2} I_{\alpha}^{2} \frac{d L_{\alpha}}{d \theta}+\frac{1}{2} I_{\beta}^{2} \frac{d L_{\beta}}{d \theta}+I_{\alpha} \frac{d \varphi_{\alpha}}{d \theta}+I_{\beta} \frac{d \varphi_{\beta}}{d \theta}$

Equations (8), (9), (10) and (12) allow us to write the following state model (13):

$$
\left(\begin{array}{c}
\frac{d I_{\alpha}}{d t} \\
\frac{d I_{\beta}}{d t} \\
\frac{d \Omega}{d t} \\
\frac{d \theta}{d t}
\end{array}\right)=\left(\begin{array}{c}
-\frac{1}{L_{\alpha}}\left(R+\frac{\partial L_{\alpha}}{\partial \theta} \Omega\right)-\frac{1}{L_{\alpha}} \frac{\partial \varphi_{\alpha}}{\partial \theta} \Omega \\
-\frac{1}{L_{\beta}}\left(R+\frac{\partial L_{\beta}}{\partial \theta} \Omega\right)-\frac{1}{L_{\beta}} \frac{\partial \varphi_{\beta}}{\partial \theta} \Omega \\
\frac{1}{J_{r}}\left(\frac{1}{2} I_{\alpha}^{2} \frac{\partial L_{\alpha}}{\partial \theta}+\frac{1}{2} I_{\beta}^{2} \frac{\partial L_{\beta}}{\partial \theta}+I_{\alpha} \frac{\partial \varphi_{\alpha}}{\partial \theta}+I_{\beta} \frac{\partial \varphi_{\beta}}{\partial \theta}-C_{r}\right) \\
\Omega \\
+\left(\begin{array}{cc}
\frac{1}{L_{\alpha}} & 0 \\
0 & \frac{1}{L_{\beta}} \\
0 & 0 \\
0 & 0
\end{array}\right)\left(\begin{array}{l}
V_{\alpha} \\
V_{\beta}
\end{array}\right)
\end{array}\right.
$$

with $\Omega$ is the rotation speed.

\section{Coupled Model}

The coupling is carried out as follows: first the air gap permeances are calculated, then the magnetic circuit is solved to find the vectors of the variations of the inductances and flux as a function of the mechanical angle. These vectors are then injected into the solver of the dynamic model. The inductances and flux values for each instant are then generated with an interpolation on the vectors considering the value of the instantaneous mechanical angle. Fig. 5 describes the magnetodynamic coupling.

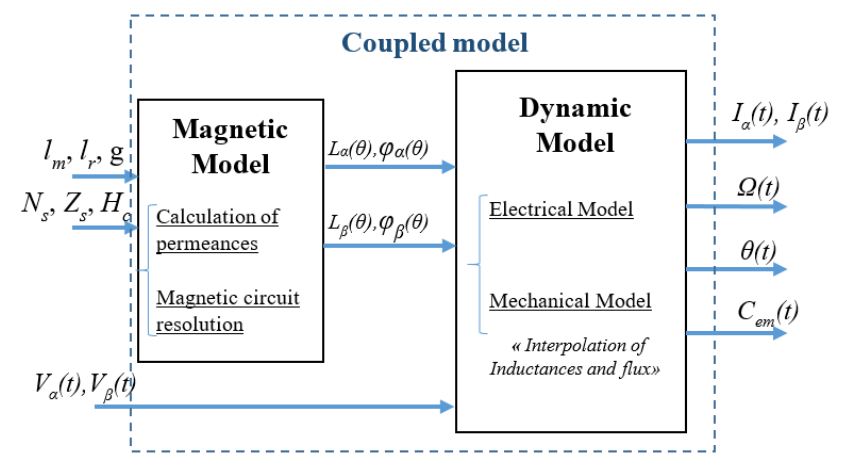

Fig. 5. Magnetodynamic Coupling.

\section{OPTIMAL CONTROL}

The motor is now in load, driving a wheel having an inertia noted $J_{\text {roue }}$ and a viscous friction coefficient, $k_{\text {roue }}$. The main purpose is to turn the wheel (initially in a position $\theta_{0}$ at time $t_{0}$ ) to reach the position $\theta_{\mathrm{f}}$ at a speed $\Omega_{\mathrm{f}}$ and in a time $\mathrm{t}_{\mathrm{f}}$ with a minimum energy. The problem could be formulated as following:

$\min _{V_{\alpha}(t), V_{\beta}(t)} \operatorname{Obj}(t)=\int_{t_{0}}^{t_{f}}\left(\left|V_{\alpha}\right|+\left|V_{\beta}\right|\right) d t$

With:

$$
\begin{gathered}
V_{\alpha \min } \leq V_{\alpha} \leq V_{\alpha \max } \\
V_{\beta \min } \leq V_{\beta} \leq V_{\beta \max } \\
\theta\left(t_{0}\right)=\theta_{0} \quad, \quad \theta\left(t_{f}\right)=\theta_{f} \\
\Omega\left(t_{0}\right)=\Omega_{0} \quad, \quad \Omega\left(t_{f}\right)=\Omega_{f} \\
I_{\alpha}\left(t_{0}\right)=I_{\alpha 0} \quad, \quad I_{\alpha}\left(t_{f}\right) \in \square \\
I_{\beta}\left(t_{0}\right)=I_{\beta 0} \quad, \quad I_{\beta}\left(t_{f}\right) \in \square
\end{gathered}
$$

In equation (14), $O b j$ represents the objective function to be minimized. This problem can be solved using the Pontryagin's maximum principle.

\section{A. Hamiltonien and Costate Vector}

The Hamiltonian of the system is given by:

$$
\begin{aligned}
& H=\left(\left|V_{\alpha}\right|+\left|V_{\beta}\right|\right)-\psi_{1} \frac{1}{L_{\alpha}}\left(R I_{\alpha}+I_{\alpha} \frac{\partial L_{\alpha}}{\partial \theta} \Omega+\frac{\partial \varphi_{\alpha}}{\partial \theta} \Omega-V_{\alpha}\right) \\
& -\psi_{2} \frac{1}{L_{\beta}}\left(R I_{\beta}+I_{\beta} \frac{\partial L_{\beta}}{\partial \theta} \Omega+\frac{\partial \varphi_{\beta}}{\partial \theta} \Omega-V_{\beta}\right) \\
& +\psi_{3} \frac{1}{J}\left(\frac{1}{2} I_{\alpha}^{2} \frac{\partial L_{\alpha}}{\partial \theta}+\frac{1}{2} I_{\beta}^{2} \frac{\partial L_{\beta}}{\partial \theta}+I_{\alpha} \frac{\partial \varphi_{\alpha}}{\partial \theta}+I_{\beta} \frac{\partial \varphi_{\beta}}{\partial \theta}-k \Omega\right) \\
& +\psi_{4} \Omega
\end{aligned}
$$


With $\psi_{1}, \psi_{2}, \psi_{3}$ and $\psi_{4}$ are costate variables, $J$ is the sum of the rotor and wheel inertia, and $k$ is the sum of the viscous friction coefficient of the rotor and the wheel.

According to the maximum principle, the costate vector must verify the following relation:

$\left(\begin{array}{c}\frac{d \psi_{1}}{d t} \\ \frac{d \psi_{2}}{d t} \\ \frac{d \psi_{3}}{d t} \\ \frac{d \psi_{4}}{d t}\end{array}\right)=\left(\begin{array}{l}-\frac{\partial H}{\partial I_{\alpha}} \\ -\frac{\partial H}{\partial I_{\beta}} \\ -\frac{\partial H}{\partial \Omega} \\ -\frac{\partial H}{\partial \theta}\end{array}\right)$

These relations give the first optimality condition.

\section{B. Optimal Control Expresssion}

The second optimality condition of the maximum principle indicates that the optimal control minimizes the Hamiltonian. Therefore, to find the command expression the sign of the functions corresponding to each command and derived from $H$ has been studied. For example, the function derived from $H$ with respect to $V_{a}$ is given by:

$H_{V \alpha}=\frac{V_{\alpha}}{\left|V_{\alpha}\right|}+\psi_{1} \frac{1}{L_{\alpha}}$

For $\psi_{l}\left(1 / L_{\alpha}\right)>0, H_{V \alpha}$ is strictly positive, then $H\left(V_{\alpha}\right)$ is strictly increasing and the minimum of the Hamiltonian is reached in $V_{\alpha}=V_{\text {min }}$. Thus, studying the sign of the derivative allowed to obtain optimal command expressions. For $V_{\text {amin }}=-$ $V_{\text {amax }}$ we get the expressions of the optimal controls $V_{\alpha}{ }^{*}$ and $V_{\beta}{ }^{*}$ are:

$V_{\alpha}^{*}=\left\{\begin{array}{cl}V_{\alpha \min } \operatorname{sign}\left(\psi_{1} \frac{1}{L_{\alpha}}\right) & \text { if }\left|\psi_{1} \frac{1}{L_{\alpha}}\right|>1 \\ 0 & \text { if }\left|\psi_{1} \frac{1}{L_{\alpha}}\right| \leq 1\end{array}\right.$

$V_{\beta}^{*}=\left\{\begin{array}{cl}V_{\beta \min } \operatorname{sign}\left(\psi_{2} \frac{1}{L_{\beta}}\right) & \text { if }\left|\psi_{2} \frac{1}{L_{\beta}}\right|>1 \\ 0 & \text { if }\left|\psi_{2} \frac{1}{L_{\beta}}\right| \leq 1\end{array}\right.$

The command with a minimum of energy for this problem involving constraints on the commands is therefore Bang-offbang type. In (18) and (19) the command is expressed in terms of the costate variables $\psi_{1}, \psi_{2}$ which in turn are expressed as a function of all the parameters of the machine according to the relation (16). The control is expressed explicitly according to all the parameters taken into account by the model.

The equations (16), (18), (19) and the machine state allow (13) us to obtain the Hamiltonian model (20):

$$
\left\{\begin{aligned}
\frac{d \psi_{1}}{d t} & =\psi_{1} \frac{1}{L_{\alpha}}\left(R+\frac{\partial L_{\alpha}}{\partial \theta} \Omega\right)-\psi_{3} \frac{1}{J}\left(I_{\alpha} \frac{\partial L_{\alpha}}{\partial \theta}+\frac{\partial \varphi_{\alpha}}{\partial \theta}\right) \\
\frac{d \psi_{2}}{d t} & =\psi_{2} \frac{1}{L_{\beta}}\left(R+\frac{\partial L_{\beta}}{\partial \theta} \Omega\right)-\psi_{3} \frac{1}{J}\left(I_{\beta} \frac{\partial L_{\beta}}{\partial \theta}+\frac{\partial \varphi_{\beta}}{\partial \theta}\right) \\
\frac{d \psi_{3}}{d t} & =\psi_{1} \frac{1}{L_{\alpha}}\left(I_{\alpha} \frac{\partial L_{\alpha}}{\partial \theta}+\frac{\partial \varphi_{\alpha}}{\partial \theta}\right)+\psi_{2} \frac{1}{L_{\beta}}\left(I_{\beta} \frac{\partial L_{\beta}}{\partial \theta}+\frac{\partial \varphi_{\beta}}{\partial \theta}\right)+\psi_{3} \frac{k}{J}-\psi_{4} \\
\frac{d \psi_{4}}{d t} & =\psi_{1} \frac{1}{L_{\alpha}} \Omega\left(-I_{\alpha} \frac{1}{L_{\alpha}}\left(\frac{\partial L_{\alpha}}{\partial \theta}\right)^{2}+I_{\alpha} \frac{\partial^{2} L_{\alpha}}{\partial \theta^{2}}-\frac{1}{L_{\alpha}} \frac{\partial L_{\alpha}}{\partial \theta} \frac{\partial \varphi_{\alpha}}{\partial \theta}+\frac{\partial^{2} \varphi_{\alpha}}{\partial \theta^{2}}\right) \\
& +\psi_{1} \frac{1}{L_{\alpha}}\left(-R I_{\alpha} \frac{1}{L_{\alpha}} \frac{\partial L_{\alpha}}{\partial \theta}+V_{\alpha}^{*} \frac{1}{L_{\alpha}} \frac{\partial L_{\alpha}}{\partial \theta}\right) \\
& +\psi_{2} \frac{1}{L_{\beta}}\left(-R I_{\beta} \frac{1}{L_{\beta}} \frac{\partial L_{\beta}}{\partial \theta}+V_{\beta}^{*} \frac{1}{L_{\beta}} \frac{\partial L_{\beta}}{\partial \theta}\right) \\
& -\psi_{3} \frac{1}{J}\left(\frac{1}{2} I_{\alpha}^{2} \frac{\partial^{2} L_{\alpha}}{\partial \theta^{2}}+\frac{1}{2} I_{\beta}^{2} \frac{\partial^{2} L_{\beta}}{\partial \theta^{2}}+I_{\alpha} \frac{\partial^{2} \varphi_{\alpha}}{\partial \theta^{2}}+I_{\beta} \frac{\partial^{2} L_{\beta}}{\partial \theta^{2}}-\frac{1}{L_{\alpha}} \frac{\partial L_{\beta}}{\partial \theta} \frac{\partial \varphi_{\beta}}{\partial \theta}+\frac{\partial^{2} \varphi_{\beta}}{\partial \theta^{2}}\right) \\
\frac{d I_{\alpha}}{d t} & =-\frac{1}{L_{\alpha}} I_{\alpha}\left(R+\frac{\partial L_{\alpha}}{\partial \theta} \Omega\right)-\frac{1}{L_{\alpha}} \frac{\partial \varphi_{\alpha}}{\partial \theta} \Omega+\frac{1}{L_{\alpha}} V_{\alpha}^{*} \\
\frac{d I_{\beta}}{d t}= & -\frac{1}{L_{\beta}} I_{\beta}\left(R+\frac{\partial L_{\beta}}{\partial \theta} \Omega\right)-\frac{1}{L_{\beta}} \frac{\partial \varphi_{\beta}}{\partial \theta} \Omega+\frac{1}{L_{\beta}} V_{\beta}^{*} \\
\frac{d \Omega}{d t}= & \frac{1}{J}\left(\frac{1}{2} I_{\alpha}^{2} \frac{\partial L_{\alpha}}{\partial \theta}+\frac{1}{2} I_{\beta}^{2} \frac{\partial L_{\beta}}{\partial \theta}+I_{\alpha} \frac{\partial \varphi_{\alpha}}{\partial \theta}+I_{\beta} \frac{\partial \varphi_{\beta}}{\partial \theta}-k \Omega\right)
\end{aligned}\right.
$$

The Hamiltonian model obtained is a two point boundary value problem. Indeed, it is necessary to find the initial conditions of the costate variables allowing bringing the system from its initial state to the desired final state. As for the final state of the current, it was left free. This implies transversality conditions on the costate vector. In fact, if the final state is free, the corresponding costate vector must be equal to zero:

$$
\left(\begin{array}{l}
\psi_{1}\left(t_{f}\right) \\
\psi_{2}\left(t_{f}\right)
\end{array}\right)=\left(\begin{array}{l}
0 \\
0
\end{array}\right)
$$

To solve this kind of problem of boundary condition type, the so-called shooting method has been used, which aims to create a function $S$ that takes as inputs the initial conditions of the variables and returns the difference between the final state obtained and the desired final state and the transversality conditions.

$$
S:\left(\begin{array}{c}
\psi_{1}\left(t_{0}\right) \\
\psi_{2}\left(t_{0}\right) \\
\psi_{3}\left(t_{0}\right) \\
\psi_{4}\left(t_{0}\right)
\end{array}\right) \rightarrow\left(\begin{array}{c}
\psi_{1}\left(t_{f}\right) \\
\psi_{2}\left(t_{f}\right) \\
\Omega\left(t_{f}\right)-\Omega_{f} \\
\theta\left(t_{f}\right)-\theta_{f}
\end{array}\right)
$$

The root of the $S$ function should be determined. This can be done with an algorithm based on the newton method, the fsolve routine of Matlab ${ }^{\circledR}$. However, one must be able to 
estimate the initial conditions to converge towards the solution. In our case, initial conditions chosen at random will give a solution to a displacement problem that we note $\mathrm{Pb}_{0}$ with an error at startup. Indeed, a differential equation solver, such as $o d e 15 s$ of Matlab $^{\circledR}$, tends to correct the initial condition error and to find a trajectory. Moreover, since the system is repetitive (step by step) the pace of the variation of the costate variables allows finding a good estimate of the initial conditions for $\mathrm{Pb}_{0}$. Fig. 6 and Fig. 7 show the variation of the costate variables for randomly chosen conditions and the estimation of the initial conditions.

The shooting method is subsequently launched with the estimated initial conditions to get the solution of the problem $P b_{0}$. From this solution, a dichotomy technique is applied to the most influential costate variables and allow us to find the solution to this displacement problem, in our case it is the variable $\psi_{4}\left(t_{0}\right)$. This solution is fast and well adapted to our study. The following problem has been considered (23).

$$
\min _{V_{\alpha}, V_{\beta}} \operatorname{Obj}(t)=\int_{0}^{1.2 s}\left(\left|V_{\alpha}\right|+\left|V_{\beta}\right|\right) d t
$$

With;

$$
\begin{gathered}
\quad-5 V \leq V_{\alpha} \leq+5 V \\
-5 V \leq V_{\beta} \leq+5 V \\
\theta\left(t_{0}\right)=0 r d \quad, \quad \theta\left(t_{f}\right)=0.5835 r d \\
\Omega\left(t_{0}\right)=0 r d / s, \quad \Omega\left(t_{f}\right)=0 r d / s \\
I_{\alpha}\left(t_{0}\right)=0 A \quad, \quad I_{\alpha}\left(t_{f}\right) \in \square \\
I_{\beta}\left(t_{0}\right)=0 A \quad, \quad I_{\beta}\left(t_{f}\right) \in \square
\end{gathered}
$$

The resolution of this problem takes about 10 minutes. Fig. 8 and Fig. 9 describe respectively the optimal commands $V_{\alpha}{ }^{*}$ and $V_{\beta}{ }^{*}$ and the corresponding switching functions.

The pulse widths gradually decrease until reaching the end position. This behavior is due to the effect of inertia, which has a great impact on energy consumption. Considering thus inertia the motor consumes less and less energy to reach the final position. Table I gives the different values of the positive and negative pulse widths of the optimal control $V_{\alpha}{ }^{*}$ of Fig. 8.

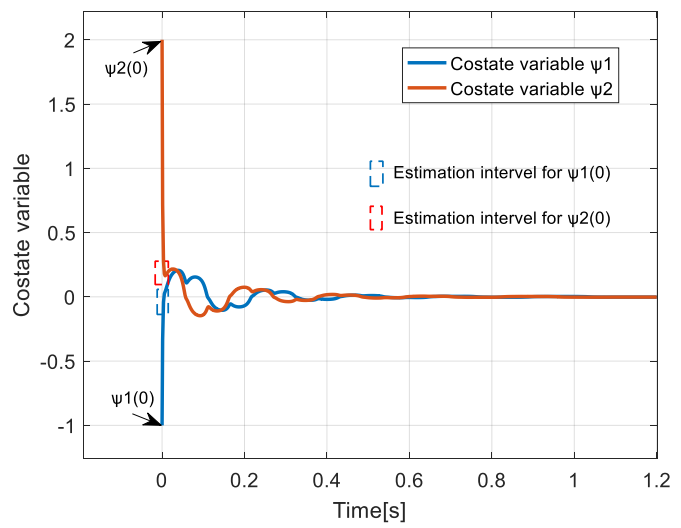

Fig. 6. Costate Variables $\psi_{1}(\mathrm{t})$ and $\psi_{2}(\mathrm{t})$ and Estimation of the Initial Conditions.

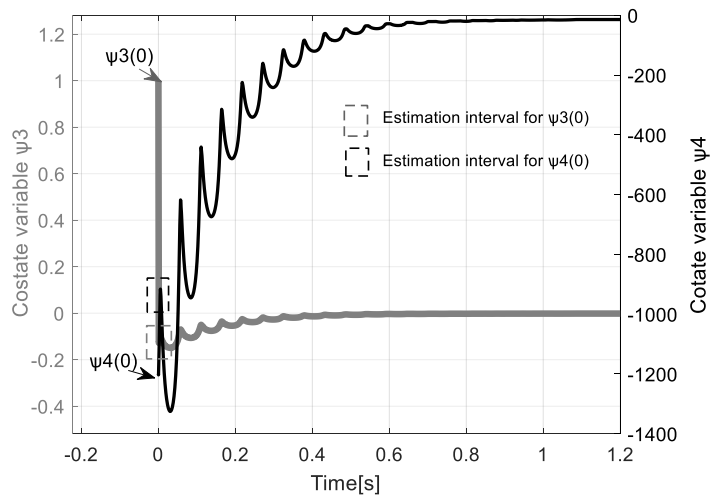

Fig. 7. Costate Variables $\psi_{3}(\mathrm{t})$ and $\psi_{4}(\mathrm{t})$ and Estimation of the Initial Conditions.

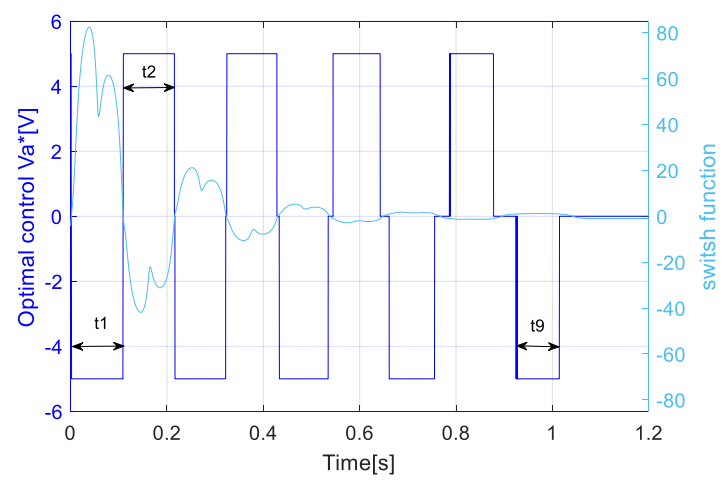

Fig. 8. Optimal Control $\mathrm{V}_{\alpha} *(\mathrm{t})$.

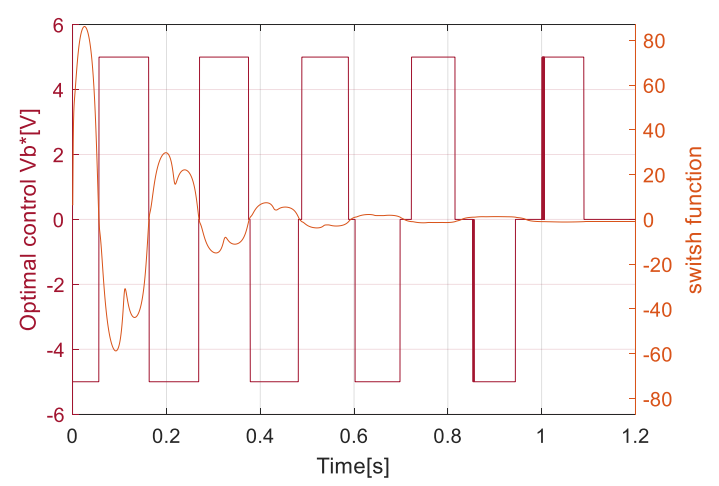

Fig. 9. Optimal Control $\mathrm{V}_{\beta} *(\mathrm{t})$.

TABLE. I. PULSE WIDTH

\begin{tabular}{|l|l|}
\hline Pulse width & Value $[\mathrm{s}]$ \\
\hline $\mathrm{t} 1$ & 0.1082 \\
\hline $\mathrm{t} 2$ & 0.1060 \\
\hline $\mathrm{t} 3$ & 0.1052 \\
\hline $\mathrm{t} 4$ & 0.1038 \\
\hline $\mathrm{t} 5$ & 0.1013 \\
\hline $\mathrm{t} 6$ & 0.0979 \\
\hline $\mathrm{t} 7$ & 0.0943 \\
\hline $\mathrm{t} 8$ & 0.0903 \\
\hline $\mathrm{t} 9$ & 0.0878 \\
\hline
\end{tabular}




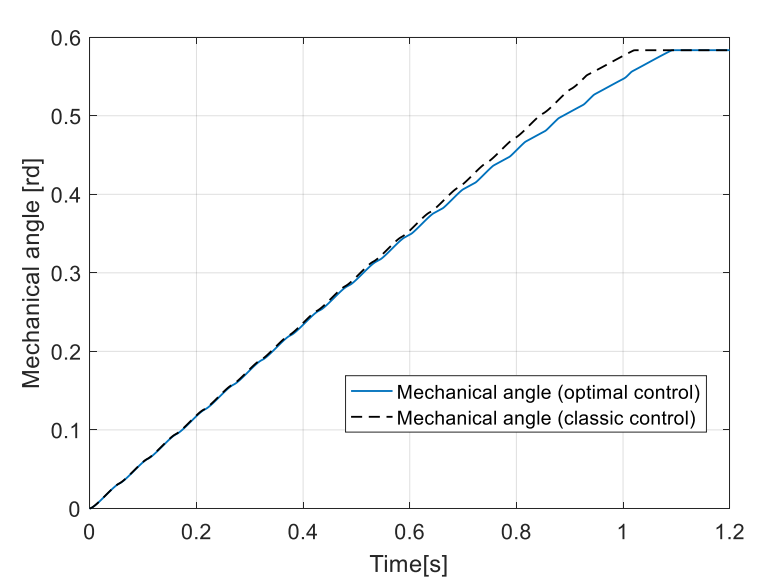

Fig. 10. Evolution of Mechanical Angle for Both Methods.

Fig. 10 shows the evolution of the mechanical angle obtained with the optimal control, in continuous line and a conventional control in dashed line. In the conventional control, the pulses have the same width, optimized to have a minimum pulse width.

The variation of the mechanical angle for the optimal control presents a curvature, which is due to the effect of the taking into account of the inertia. For the classic control, the position is reached more quickly but the motor in this case consume more energy. The obtained optimal control offers a gain of absorbed power of $5,6 \%$.

Fig. 11 presents the evolution of the costate variables $\psi_{3}(t)$ and $\psi_{4}(t)$ related respectively to the speed $\Omega(t)$ and to the position $\theta(\mathrm{t})$. The costate variables $\psi_{1}(t)$ and $\psi_{2}(t)$ will have the same pace as the switch functions with a difference in amplitude due to the terms $1 / L_{\alpha}$ and $1 / L_{\beta}$ in (8) and (9).

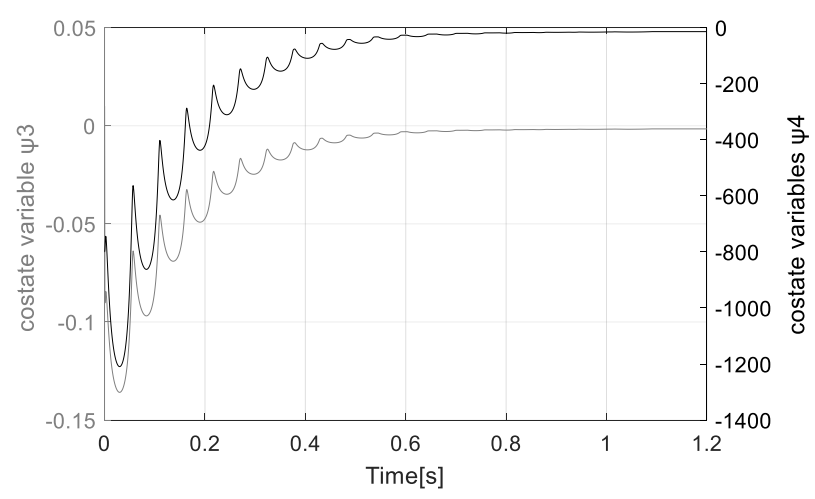

Fig. 11. Costate Variables $\psi 3(\mathrm{t})$ and $\psi 4(\mathrm{t})$.

\section{Global OPTIMIZATION APPROACH}

The application of the Pontryagin's maximum principle gives a formulation that allows the automatic generation of the optimal control through costate equations. This optimal control takes into account all input machine dynamic model parameters. Therefore, it is possible to vary the design inputs of the machine and find the optimal control for each geometric configuration. The idea is to add an optimization loop on design parameters. This will give an overall optimization approach to design and control.

\section{A. Global Model and Optimisation Loop}

Fig. 12 describes the interaction between the different models, the dichotomy approach and the optimization loop whenever both the rotor rings length and the magnet length should be opimized. The magnetic model is now coupled to the Hamiltonian model. The dichotomy method allows finding initial conditions of the costate model. Then an optimization loop is applied on the design parameters.

\section{B. Effet De La Longeur De L'aimant Et Des Coronnes}

In this section, the influence of the rings and magnet length on both the energetic performances machine and on the optimal control has been studied.

Fig. 13 describes a section of the rotor and the two design parameters that we study.

An unloaded machine has been simulated for a 5 steps displacement, i.e. $\theta\left(t_{f}\right)=0.157[\mathrm{rd}]$, and a final time $t_{f}=0.1 \mathrm{~s}$. The objective function and the constraints on the controls, speed and currents have been kept as in the equation (23). However, we vary the length of the crown between $8 \mathrm{~mm}$ and $22 \mathrm{~mm}$, and the magnet length between $0.5 \mathrm{~mm}$ and $1.6 \mathrm{~mm}$. An objective function denoted $O b j$ ' is defined such that:

$$
\operatorname{Obj}^{\prime}(t)=\int_{0}^{0.1 s}\left(V_{\alpha}^{2}(t)+V_{\beta}^{2}(t)\right) d t
$$

Minimizing the objective function $O b j$ ' is the minimization of the Obj function. The only difference is that the Obj' function would have a smoother evolution and would be more efficient we use a gradient descent.

Fig. 14 describes the evolution of the function $O b j$ ' as a function of $l_{m}$ and $l_{r}$.

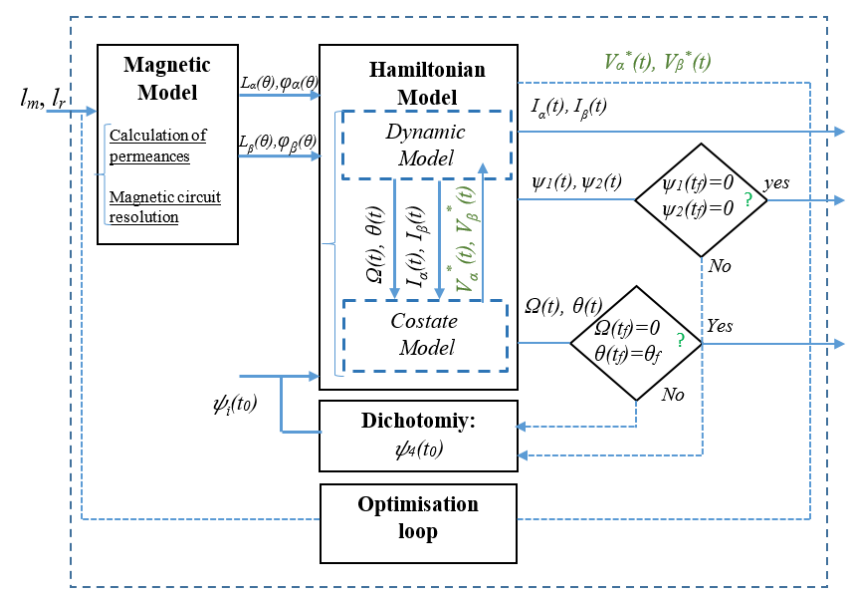

Fig. 12. Resolution Process.

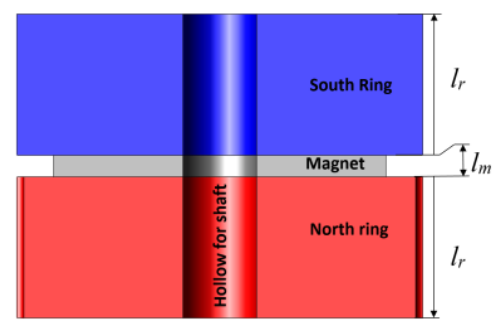

Fig. 13. Rotor Section. 


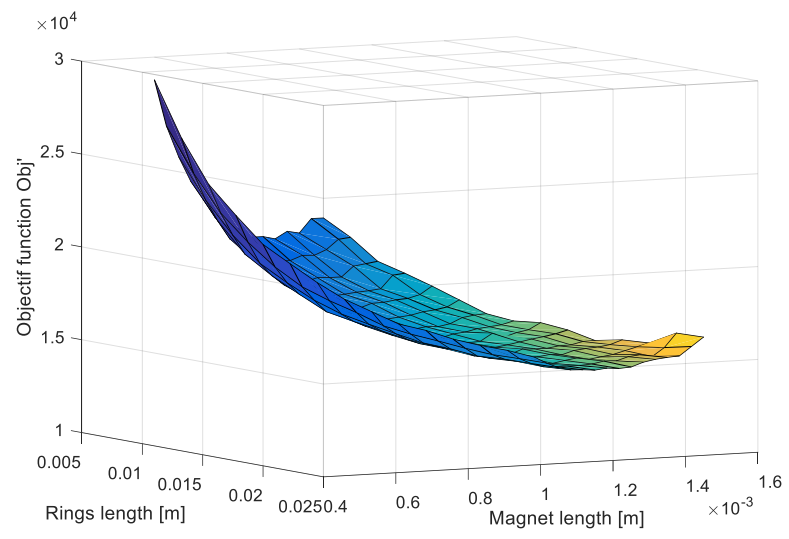

Fig. 14. Evolution of Objective Function Obj.

The evolution of the surface is inversely proportional to the magnet thickness. This is explained by the fact that a thicker magnet provides a larger magnetomotive force and thus the machine needs less energy to turn.

In the direction of the increase of the length of the crowns, the surface is decreasing then increasing this is explained by two phenomena. Taking the cases where $l_{m}=0.5 \mathrm{~mm}$, there exists a value of $l_{r}$ that we note $l_{r}^{*}$ for which the function $O b j$, is minimal. From this value if the value of $l_{r}$ is decreased there will be less interaction between the rotor and the stator and therefore the motor will need more energy to turn. In the other hand, if from $l_{r}^{*}$, the value of $l_{r}$ is increased, the inertia of the rotor increases and the motor will need more energy to turn. So for each value of the magnet thickness there exists a value of $1 \mathrm{~m}$ for which the $O b j$ ' function is minimal. This minimum presents a compromise between rotor-stator interaction and rotor inertia. Fig. 15 describes the evolution of optimal control $V_{\beta}{ }^{*}$ as a function of $l_{r}$ Note that the pulse widths are minimal for the value $l_{r}^{*}$ which explains the behavior of Obj'.

\section{Global Optimisation Problem}

The purpose here is to find the optimal values of $l_{r}$ and $l_{m}$ that minimize the consumption consideration that minimizing $O b j$ ' is minimizing Obj. The global optimization problem could be written as follows:

$$
\min _{l_{m}, l_{r}}\left(\min _{V_{\alpha}(t), V_{\beta}(t)}\left(\int_{0}^{0.1 s}\left(\left|V_{\alpha}\right|+\left|V_{\beta}\right|\right) d t\right)\right)
$$

with:

$$
\begin{gathered}
8 m m \leq l_{r} \leq 22 \mathrm{~mm} \\
0.5 \mathrm{~mm} \leq l_{m} \leq 1.6 \mathrm{~mm} \\
-5 \mathrm{~V} \leq V_{\alpha} \leq+5 \mathrm{~V} \\
-5 \mathrm{~V} \leq V_{\beta} \leq+5 \mathrm{~V} \\
\theta\left(t_{0}\right)=0 \mathrm{rd} \quad, \quad \theta\left(t_{f}\right)=0.157 \mathrm{rd} \\
\Omega\left(t_{0}\right)=0 \mathrm{rd} / \mathrm{s} \quad, \quad \Omega\left(t_{f}\right)=0 \mathrm{rd} / \mathrm{s} \\
I_{\alpha}\left(t_{0}\right)=0 \mathrm{~A} \quad, \quad I_{\alpha}\left(t_{f}\right) \in \square \\
I_{\beta}\left(t_{0}\right)=0 \mathrm{~A} \quad, \quad I_{\beta}\left(t_{f}\right) \in \square
\end{gathered}
$$

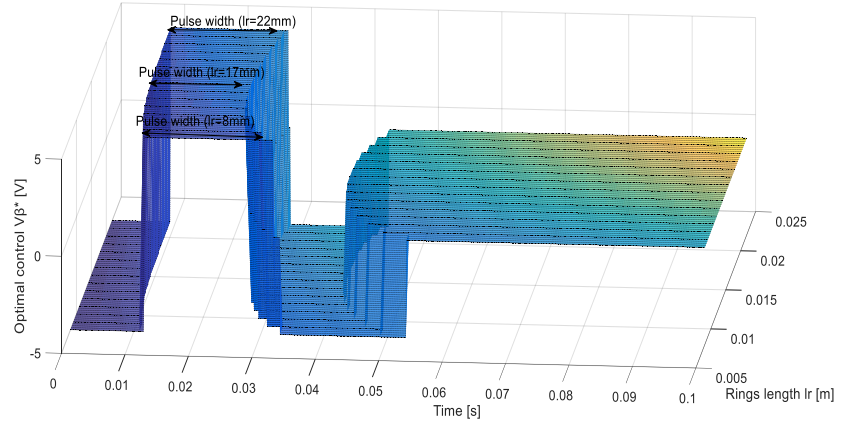

Fig. 15. Evolution of Optimal Control $\mathrm{V}_{\beta}{ }^{*}(\mathrm{t})$.

The optimization loop is launched on the design with the fmincon routine of Matlab ${ }^{\circledR}$. Table II shows the initial and the optimal sizing.

Table III illustrates the objective function and the input power for both configurations.

The optimal sizing provides a gain of $34.6 \%$ for input power compared to the initial configuration. The obtained gain is significant mainly because of the magnet length that has increased.

TABLE. II. INITIAL AND OPTIMAL SIZING

\begin{tabular}{|l|l|l|}
\hline & Initial sizing & Optimal sizing \\
\hline Length of rings $[\mathrm{mm}]$ & 13 & 11.7 \\
\hline Length of magnet $[\mathrm{mm}]$ & 1 & 1.6 \\
\hline
\end{tabular}

TABLE. III. OBJECTIVE FUNCTION AND INPUT POWER

\begin{tabular}{|l|l|l|}
\hline & Objective function & Input power $[\mathbf{w}]$ \\
\hline Initial sizing & 13250 & 5.43 \\
\hline Optimal sizing & 10650 & 3.55 \\
\hline
\end{tabular}

\section{CONCLUSION}

The main contribution of this paper is to obtain in the same process, the minimization of the energy by optimal control and the minimization of the energy by optimal sizing. First, a magnetodynamic model based on a permeances network was developed. Then the Pontryagin Maximum Principle was applied to the magnetodynamic model in order to find the optimal control minimizing the energy. The application of the PMP allows us to explicitly express the command according to all model parameters and to have a Hamiltonian model that automatically generates optimal control. The study has shown that boundary value problem encountered at the resolution level of the Hamiltonian model can be solved by a simple dichotomy when it is a control problem of an electric machine. The results showed a gain of $5.7 \%$ compared to a conventional control for a given positioning problem. Finally, adding an optimization loop on design inputs to give an overall optimization approach. 


\section{REFERENCES}

[1] G. Lei, J. Zhu, and Y. Guo "Multydisciplinary Design Optimisation Methods for Electrical Machines and Drive Systems", Springer-Verlag Berlin Heidelberg, 2016.

[2] D. Dorrell "A Review of the Methods for improving the Efficiency of Drive Motors to Meet IE4 Efficiency Standards", Journal of power electronics, pp. 842-851, September 2014.

[3] H. Alihalli, M. Ilyas Bayindir, "Time-energy optimal control of vector controlled induction motor", COMPEL: Int. J. Computation and Mathematics in Electrical and Electronic Engineering, pp. 235-251, 2002.

[4] V. P. Lapshin, I. A. Turkin and V. V. Khristoforova, "Synthesis of Electromechanical Position Control System by Means of Maximum Principle," 2018 International Russian Automation Conference (RusAutoCon), Sochi , pp. 1-4, 2018.

[5] C. M. Vega, J. R. Arribas and J. Herrero, "Optimal-time control of squirrel cage induction motors with constant load torque," IEEE 2002 28th Annual Conference of the Industrial Electronics Society. IECON 02, Sevilla, pp. 2039-2044 vol.3, 2002.

[6] R. Zheng, R. Cai and M. Li, "Energy management of electric vehicles with permanent magnet synchronous in-wheel motors using pontryagin's minimum principle," IECON 2017 - 43rd Annual Conference of the IEEE Industrial Electronics Society, Beijing, pp. 2275-2280, 2017.

[7] A. S. Revko and R. D. Yershov, "Control Rapidity Optimization Technique of DC-Motor Driven by Quasi-Resonant Converter Using Pontryagin's Maximum Principle," 2018 IEEE 38th International Conference on Electronics and Nanotechnology (ELNANO), Kievpp. 705-710, , 2018.

[8] L. Roubache, K. Boughrara, F. Dubas and R. Ibtiouen, "New Subdomain Technique for Electromagnetic Performances Calculation in Radial-Flux Electrical Machines Considering Finite Soft-Magnetic Material Permeability", in IEEE Transactions on Magnetics, vol. 54, no. 4, pp. 1-15, April 2018.

[9] Z. Djelloul-Khedda, K. Boughrara, F. Dubas and R. Ibtiouen, "Nonlinear Analytical Prediction of Magnetic Field and Electromagnetic Performances in Switched Reluctance Machines," in IEEE Transactions on Magnetics, vol. 53, no. 7, pp. 1-11, July 2017.

[10] M. H. Mohammadi, R. C. P. Silva and D. A. Lowther, "Finding Optimal Performance Indices of Synchronous AC Motors," in IEEE Transactions on Magnetics, vol. 53, no. 6, pp. 1-4, June 2017.
[11] M. H. Mohammadi, R. C. P. Silva, D. A. Lowther,'Incorporating Control Strategies Into the Optimization of Synchronous AC Machines: A comparison of Methodologies", IEEE Transaction on magnetics, vol. 54, no. 3, March 2018.

[12] H. Geering, "Optimal Control with Engineering Application", IEEE Control Systems, vol. 31, no. 5, pp. 115-117, 2011.

[13] Zhu, J. Trélat, E. \& Cerf, M. Pac," Geometric optimal control and applications to aerospace", J. Math. Ind, 2198-4115, 2017.

[14] S. Uebel, N. Murgovski, C. Tempelhahn and B. Bäker, "Optimal Energy Management and Velocity Control of Hybrid Electric Vehicles," in IEEE Transactions on Vehicular Technology, vol. 67, no. 1, pp. 327337, Jan. 2018.

[15] N. Kim, S. Cha and H. Peng, "Optimal Control of Hybrid Electric Vehicles Based on Pontryagin's Minimum Principle," in IEEE Transactions on Control Systems Technology, vol. 19, no. 5, pp. 12791287, Sept. 2011.

[16] H. Dagdougui, "Optimal Control of a Network of Power Microgrids Using the Pontryagin's Minimum Principle", IEEE Transactions on Control Systems Technology, vol. 22, no. 5, pp. 1942-1948, 2014.

[17] M. Matsuri, M. Nakamura, T. Kosaka, "Instantaneous torque analysis of hybrid stepping motor," IEEE Transactions on Industry Applications, vol. 34, no 5 , pp. 1176-1182, 1996.

[18] C. Kuert, M. Jufer and Y. Perriard, "New method for dynamic modeling of hybrid stepping motors," Conference Record of the 2002 IEEE Industry Applications Conference. 37th IAS Annual Meeting (Cat. No.02CH37344), Pittsburgh, PA, pp. 6-12 vol.1, USA, 2002.

[19] P. P. Acarnely, "Stepping Motors: A Guide to Theory and Practice $4^{\text {th }}$ edition ", IET Control Engineering Series 63, London, 2007.

[20] I. Ionica, M. Modereanu, A. Morega, C Boboc, "Design and modeling of a hybrid stepper motor", IEEE International Symposium on Advanced Topics in Electrical Engineering, pp. 192-195, 2017.

[21] C. Stuebig, B. Ponick, "Comparision of Calculation Methods for Hybrid Stepping Motors", IEEE Transaction on Industry Applications, Vol. 48, noㅇ, pp2182-2189, Nov-Dec 2012.

[22] W. Bêkir, L. E. Amraoui and F. Gillon, "Dynamic performances determination of a HSM using a finite element validation of the magnetostatic model", 2016 International Conference on Electrical Sciences and Technologies in Maghreb (CISTEM), pp. 1-6, Marrakech, 2016. 